АНАЛИЗ ФАКТИЧЕСКОГО ПИТАНИЯ ЖЕНСКОГО НАСЕЛЕНИЯ НОВОСИБИРСКА

В ЗАВИСИМОСТИ ОТ ВЕЛИЧИНЫ ГЛИКЕМИЧЕСКОГО ИНДЕКСА ИХ РАЦИОНА

(с) А.К. Кунцевич1* , С.В. Мустафина', Е.Г. Веревкин ${ }^{1,2}$, Л.В. Щербакова', О.Д. Рымар ${ }^{1}$

\begin{abstract}
' Научно-исследовательский институт терапии и профилактической медицины. Филиал государственного бюджетного научного учреждения «Федеральный исследовательский центр Институт цитологии и генетики Сибирского отделения Российской академии наук», Новосибирск, Россия

2 ФГБНУ « Научно-исследовательский институт молекулярной биологии и биофизики», Новосибирск, Россия
\end{abstract}

ВВЕДЕНИЕ. При выборе продуктов питания, богатых углеводами, важно учитывать не только их химический состав, но также способность влиять на уровень постпрандиальной глюкозы крови (гликемический ответ). Качественным показателем такой способности является гликемический индекс (ГИ) углеводсодержащего продукта. В последнее время появились определенные свидетельства, что контроль за ГИ питания может быть важным для профилактики и диетологической терапии нарушений метаболизма.

ЦЕЛЬ. Определение величины ГИ фактического питания у женщин г. Новосибирска и изучение связи с нарушением метаболического обмена и ожирением.

МЕТОДЫ. Работа выполнена по дизайну «одномоментное популяционное исследование» в рамках международного проекта HAPIEE на случайной репрезентативной выборке неорганизованного населения г. Новосибирска, 4397 женщин, средний возраст 57,7 года, без сахарного диабета 2 типа (СД2). Для расчета ГИ питания использовали данные Международной таблицы гликемического индекса и гликемической нагрузки (2008). Для оценки фактического питания использовали частотный метод. Критерии метаболического синдрома (MC) использовали в соответствии с Российскими национальными рекомендациями BHOK (2009), а также международными рекомендациями NCEP ATP III (2001) и JIS (2009). Статистическая обработка данных проведена с использованием пакета прикладных программ SPSS 13.0 - критерий Бонферрони в процедуре GLM (Общие линейные модели). Оценку отношения шансов (OR) проводили с использованием бинарной логистической регрессии в квартилях ГИ. Различия считались статистически значимыми при $\mathrm{p}<0,05$.

РЕЗУЛЬтАТЫ. Величина ГИ питания женщин была в среднем низкой и составила 53,1 единицы. В квартилях ГИ отношение шансов развития МС по всем использованным критериям значимо не различалось. ГИ у женщин в группе

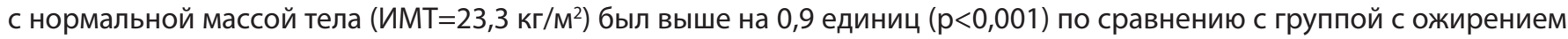
$($ ИМТ=37,3 кг/м²). Питание было несбалансированным и не соответствовало рекомендациям во всех группах исследованных женщин. Было отмечено снижение в группе с ожирением в 1,6 раза величины энергоценности питания в расчете на 1 кг массы тела.

ЗАКЛЮЧЕНИЕ. Питание женщин г. Новосибирска является несбалансированным, с повышенным потреблением жиров и недостаточным потреблением углеводов. Величина ГИ является близкой к ГИ питания населения европейских стран. Наличие связи ГИ питания женщин с нарушением метаболизма в исследованной выборке населения не установлено.

КЛЮЧЕВЫЕ СЛОВА: питание, гликемический индекс, метаболический синдром, ожирение.

\title{
ANALYSIS OF THE ACTUAL NUTRITION OF THE FEMALE POPULATION OF NOVOSIBIRSK, DEPENDING ON THE MAGNITUDE OF THE GLYCEMIC INDEX OF THEIR DIET
}

\author{
(c) Alexander K. Kuntsevich ${ }^{1 *}$, Svetlana V. Mustafina', Evgeniy G. Verevkin,2, Liliya V. Shcherbakova' , Oksana D. Rymar ${ }^{1}$
}

${ }^{1}$ Research Institute of Internal and Preventive Medicine - Branch of the Institute of Cytology and Genetics, Novosibirsk, Russia 2Institute of Molecular Biology and Biophysics, Novosibirsk, Russia.

BACKGROUND. When choosing foods rich in carbohydrates, it is important to consider not only their chemical composition, but also the ability to influence the level of postprandial blood glucose (glycemic response). A qualitative indicator of this ability is the glycemic index (Gl) of a carbohydrate-containing product. Recently, there have been some evidences that control of GI nutrition can be important for prevention and nutrition therapy of metabolic disorders.

AIM. To determine of the magnitude of the glycemic index (GI) of actual nutrition in women in Novosibirsk, and the study of the connection with metabolism and obesity.

MATERIAL AND METHODS. The study was designed as cross-sectional research and was carried out within the framework of the international HAPIEE project on a random representative sample of the unorganized population of Novosibirsk, 4397 
women, the average age was $57.7 \pm 7.1$, without diabetes. For the calculation of Gl nutrition, the International Table of Glycemic Index and Glycemic Load (2008) were used. The frequency method was used to evaluate the actual nutrition. Metabolic Syndrome (MS) criteria were used in accordance with the Russian National Recommendations of the GEF (2009), as well as with the international recommendations of NCEP ATP III (2001) and JIS (2009). Statistical processing of data was carried out using the SPSS 13.0 software package (Bonferroni criterion in the GLM procedure). The odds ratio (OR) was estimated using binary logistic regression in quartiles of the glycemic index. Differences were considered statistically significant at $p<0.05$.

RESULTS. The GI of women's nutrition was on average low and amounted to 53.1 units. In the quartiles of the GI OR, the MS for all the criteria used was not significantly different. In the quartiles of the body mass index (BMI) in women with Gl in the group with normal body weight $\left(\mathrm{BMI}=23.3 \mathrm{~kg} / \mathrm{m}^{2}\right)$ was significantly higher by 0.9 units $(\mathrm{p}<0.001)$ compared with the obese group $\left(\mathrm{BMI}=37.3 \mathrm{~kg} / \mathrm{m}^{2}\right)$. The diet was unbalanced and did not meet the recommendation in all groups of women studied. It was noted a significant reduction in the group with obesity 1.6 times the value of total caloric intake per $1 \mathrm{~kg}$ of body weight.

CONCLUSIONS. Nutrition of women in Novosibirsk is unbalanced, with increased consumption of fat and inadequate intake of carbohydrates. The Gl value is close to the GI nutrition of the population of European countries. The presence of a connection between the GI nutrition of women with a metabolic disorder in the sample of the population was not established.

KEYWORDS: nutrition, glycemic index, metabolic syndrome, obesity.

Установление факта, что уровень постпрандиальной гликемии различается в зависимости от того, в форме каких продуктов и блюд человек потребляет углеводы, привело к понятию гликемического индекса (ГИ) для классификации углеводсодержащих продуктов. ГИ является показателем скорости усвоения углеводов и характеризует способность данных углеводов при попадании с пищей в организм повышать уровень глюкозы крови в сравнении со стандартом, как правило, с глюкозой или белым хлебом [1]. Данные ГИ различных продуктов приведены в Международных таблицах гликемического индекса и гликемической нагрузки [2], а также на сайте www.glycemicindex.com.

При очевидной необходимости широкого подхода к лечению и профилактике ожирения - оптимизации двигательного режима и коррекции рациона питания, с учетом влияния среды и возрастающей урбанизации, а также роли государственной поддержки в отношении повышения физической активности населения [3], вероятно, что контроль за ГИ питания населения может быть важным профилактическим фактором [4]. По данным скрининга 2003-2005 гг., распространенность ожирения в сибирской популяции в возрасте 45-69 лет у женщин составила 47\%, среднее значение ИМТ - 30,2 кг/м² [5], что подчеркивает важность оценки факторов питания, влияющих на метаболический обмен. В ряде популяционных исследований установлено наличие связи питания, а также отдельных компонентов рациона с риском развития метаболического синдрома (MC) у населения [6]. Кроме того, появились свидетельства, что контроль за потреблением продуктов с высоким ГИ, а также замена их на продукты с низким ГИ могут быть полезными для профилактики и диетологической терапии нарушений метаболизма $[7,8]$. Результаты мета-анализа проспективных исследований (37 работ, население США, Канады, Австралии и Европы) по связи ГИ питания с риском развития хронических заболеваний показали наличие значимой связи между ГИ пищи и риском развития таких заболеваний, как СД2, сердечно-сосудистые заболевания, заболевания желчного пузыря и рака молочной железы [9]. Данные о связи ГИ питания с МС немногочисленны. Было установлено в одномоментном популяционном исследовании населения США наличие значимой прямой связи величины ГИ с распространенностью МС в квинтилях ГИ [10].

\section{ЦЕЛЬ}

Целью нашей работы явились изучение ГИ фактического рациона питания у женщин г. Новосибирска (45-69 лет) и оценка наличия возможной связи с метаболическими нарушениями.

\section{МАТЕРИАЛЫ И МЕТОДЫ}

Данная работа проведена по дизайну «одномоментное популяционное исследование». Использованы материалы международного проекта HAPIEE («Детерминанты сердечно-сосудистых заболеваний в Восточной Европе: многоцентровое когортное исследование», принципиальные исследователи в Новосибирском центре - проф. Малютина С.К., акад. РАН Никитин Ю.П.). Проект НАРІЕЕ поддержан грантами Wellcome Trust, UK 064947/Z/01/Z;081081/Z/06/Z; National Institute of Aging, USA (1R01 AG23522). Наше исследование включало оценку фактического питания и изучение возможной связи величины ГИ углеводов продуктов питания с дефинициями риска МС. Случайная репрезентативная выборка неорганизованного населения г. Новосибирска включала 9360 жителей (мужчины и женщины, 45-69 лет). В данное исследование были включены 4397 женщин, средний возраст 57,7 лет, без СД2.

Для оценки питания использовали адаптированный вопросник по оценке частоты потребления пищевых продуктов (Brunner E. et al., 2001; Мартинчик А.Н. и др., 1998). Вопросник заполнялся специально подготовленным интервьюером со слов участника обследования и включал 142 продукта питания.

База химического состава продуктов питания, включенных в вопросник, составлена на основании справочников «Таблицы химического состава и калорийности российских продуктов питания» (2007) и «Химический состав пищевых продуктов» (1987).

Для расчета ГИ рационов использовали данные Международных таблиц гликемического индекса и гликемической нагрузки [2], а также данные, опубликованные Лобыкиной Е.Н., Колтуном В.С̧., Хвостовой О.И. [4]. 
Величину гликемического индекса диеты рассчитывали по формуле:

ГИ диеты $=\Sigma$ (ГИ продукта $\times$ количество углеводов в продукте) / общее количество углеводов в диете [11].

Критерии МС принимались в соответствии с Российскими национальными рекомендациями BHOK [12]. Для сравнения использовали критерии NCEP ATP III (2001) [13] и согласованные критерии JIS (2009) [14].

Статистическая обработка данных проведена с использованием пакета прикладных программ SPSS 13.0 критерий Бонферрони в процедуре GLM (Общие линейные модели). Сравнение частот качественных признаков проводилось с помощью критерия X2. Оценку отношения шансов проводили с использованием бинарной логистической регрессии. Результаты представлены как среднее \pm стандартное отклонение $(\mathrm{M} \pm \mathrm{SD})$. Различия считались статистически значимыми при $\mathrm{p}<0,05$.

\section{РЕЗУЛЬТАТЫ И ОБСУЖДЕНИЕ}

Среднее значение ГИ питания у женщин составило $53,1 \pm 3,9$, которое можно принять как низкое, исходя из критериев классификации ГИ: высокий уровень более 70 единиц, средний - 56-69 и низкий - менее 55 [2]. Также значение ГИ близко к популяционным данным ГИ питания женщин в западных странах - Италии (ГИ=51,7; $\mathrm{n}=4242)$ [15], Испании (ГИ=55,6; $\mathrm{n}=4001)$ [16], США (ГИ=54,1; $n=18341)$ [17]. В странах с традиционно высоким потреблением риса ГИ питания был выше. Так, у женщин Японии среднее значение ГИ составило $68,0$ единиц ( $n=1598)$ [18], у женщин Китая - 70,0 единиц $(n=73328)[19]$.

Из таблицы 1 видно, что величина окружности талии во всех квартилях ГИ была выше рекомендованной нормы. Также во всех квартилях наблюдалась избыточная масса тела (ИМТ был $>25$ кг/м²). У женщин в 4 квартиле ГИ величина ИМТ была значимо ниже по сравнению с 1 квартилем. По массе тела у женщин различие между крайними квартилями было значимо и составило соответственно 76,5 кг и 72,9 кг. Средний возраст у женщин в 1 квартиле ГИ был значимо ниже по сравнению с 4 квартилем, соответственно 57,1 и 58,6 лет ( $<<0,001$ ) (табл. 1).

Триглицериды крови (ТГ) у женщин соответствовали

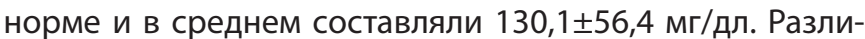
чий между крайними квартилями ГИ по этому показателю не было. Также не наблюдалось значимого различия величины холестерина липопротеидов высокой плотности (ХС ЛПВП) между квартилями ГИ, при этом величина ХС ЛПВП в каждом квартиле ГИ была в норме (>50 мг/дл). Концентрация холестерина липопротеидов низкой плотности (ХС ЛПНП) у женщин в среднем соста-

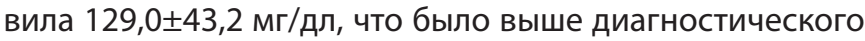
критерия, при отсутствии значимого различия между квартилями ГИ.

Значимое различие между квартилями ГИ у женщин наблюдалось только по такому показателю как уровень глюкозы плазмы крови натощак, хотя это различие было минимальным - около 1\%. Значение глюкозы в каждом квартиле ГИ было в пределах нормы, среднее значение

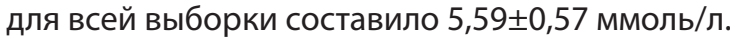

Во всех квартилях ГИ систолическое артериальное давление (САД) крови было выше нормы, отсутствовали значимые различия между крайними квартилями. Также величина диастолического артериального давления (ДАД) была выше нормы и значимо не различалась между квартилями ГИ.

Показатель общего холестерина крови (ОХC) у женщин в выборке составил 249 48 мг/дл, что выше нормы, при этом между крайними квартилями ГИ значимого различия не было (см. табл. 1).

Средняя энергоценность (калорийность) рациона (ЭЦ) в 1 квартиле ГИ была несколько выше (на 3\%) по сравнению с 4 квартилем. Но при расчете величины ЭЦ на 1 кг массы тела этот показатель между 1 и 4 квартилями ГИ значимо не различался. В минимальном квартиле ГИ доля белков - 14,3\% и жиров - 46,5\% были значимо

Таблица 1. Антропометрические и биохимические показатели участников (женщины) исследования в квартилях ГИ (M \pm SD)

\begin{tabular}{|c|c|c|c|c|c|c|c|}
\hline \multirow{3}{*}{ Показатели } & \multicolumn{4}{|c|}{ Квартили ГИ } & \multicolumn{3}{|c|}{$\mathbf{P}$} \\
\hline & 1 & 2 & 3 & 4 & $1-2$ & $1-3$ & $1-4$ \\
\hline & $n=1099$ & $n=1099$ & $n=1100$ & $n=1099$ & & & \\
\hline ГИ & $47,9 \pm 2,4$ & $52,2 \pm 0,8$ & $54,6 \pm 0,7$ & $57,7 \pm 1,4$ & $<0,001$ & $<0,001$ & $<0,001$ \\
\hline $\min -\max \Gamma И$ & $34,33-50,74$ & $50,74-53,48$ & $53,48-55,84$ & $55,84-65,69$ & & & \\
\hline Возраст, лет & $57,1 \pm 7,0$ & $57,4 \pm 7,0$ & $58,0 \pm 7,1$ & $58,6 \pm 7,3$ & 1,00 & $<0,09$ & $<0,001$ \\
\hline Масса тела, кг & $76,5 \pm 14,5$ & $75,1 \pm 14,5$ & $74,3 \pm 14,4$ & $72,9 \pm 14,5$ & 0,126 & 0,002 & $<0,001$ \\
\hline Окружность талии, см & $91,3 \pm 13,0$ & $90,8 \pm 12,6$ & $90,7 \pm 12,9$ & $90,2 \pm 13,2$ & 1,00 & 0,024 & 0,000 \\
\hline 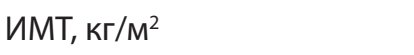 & $30,3 \pm 5,7$ & $30,0 \pm 5,6$ & $29,6 \pm 5,6$ & $29,3 \pm 5,6$ & 0,001 & $<0,001$ & $<0,001$ \\
\hline ОХС крови, мг/дл & $248 \pm 46$ & $249 \pm 47$ & $251 \pm 49$ & $249 \pm 50$ & 1,00 & 0,665 & 1,00 \\
\hline ХС ЛПВП, мг/дл & $61,5 \pm 18,4$ & $60,6 \pm 13,0$ & $61,0 \pm 13,6$ & $61,2 \pm 13,7$ & 1,00 & 0,035 & 1,00 \\
\hline ТГ крови, мг/дл & $129 \pm 54$ & $131 \pm 58$ & $130 \pm 56$ & $130 \pm 57$ & 1,00 & 1,00 & 1,00 \\
\hline ХС ЛПНП, мг/дл & $127 \pm 43$ & $129 \pm 43$ & $131 \pm 43$ & $129 \pm 43$ & 1,00 & 0,474 & 1,00 \\
\hline Глюкоза натощак, ммоль/л & $5,64 \pm 0,58$ & $5,59 \pm 0,55$ & $5,56 \pm 0,56$ & $5,57 \pm 0,60$ & 0,134 & 0,002 & 0,016 \\
\hline САД, мм рт. ст. & $143 \pm 36$ & $142 \pm 26$ & $145 \pm 26$ & $145 \pm 27$ & 1,00 & 0,632 & 0,160 \\
\hline ДАД, мм рт. ст. & $91,0 \pm 31$ & $89,8 \pm 14$ & $90,3 \pm 13$ & $90,3 \pm 14$ & 0,941 & 1,00 & 1,00 \\
\hline
\end{tabular}




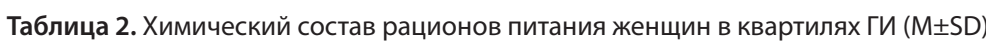

\begin{tabular}{|c|c|c|c|c|c|c|c|}
\hline \multirow{3}{*}{ Показатели } & \multicolumn{4}{|c|}{ Квартили ГИ } & \multicolumn{3}{|c|}{$\mathbf{P}$} \\
\hline & 1 & 2 & 3 & 4 & $1-2$ & $1-3$ & $1-4$ \\
\hline & $n=1099$ & $n=1099$ & $n=1100$ & $n=1099$ & & & \\
\hline ЭЦ, ккал/сут & $2304 \pm 719$ & $2446 \pm 728$ & $2352 \pm 652$ & $2225 \pm 577$ & $<0,001$ & 0,568 & 0,035 \\
\hline ЭЦ/кг массы тела & $31,1 \pm 11,2$ & $33,7 \pm 12,0$ & $32,9 \pm 11,4$ & $31,7 \pm 10,5$ & $<0,001$ & 0,001 & 1,00 \\
\hline Белки, г/сут & $90,3 \pm 32,2$ & $91,7 \pm 29,9$ & $85,0 \pm 26,1$ & $77,9 \pm 24,4$ & 1,00 & $<0,001$ & $<0,001$ \\
\hline Жиры, г/сут & $118,2 \pm 41$ & $122,1 \pm 41$ & $114,0 \pm 37$ & $103,6 \pm 33$ & 0,101 & 0,064 & $<0,001$ \\
\hline Холестерин пищи, мг/сут & $337 \pm 152$ & $360 \pm 155$ & $333 \pm 160$ & $300 \pm 143$ & 0,002 & 1,00 & $<0,001$ \\
\hline Углеводы, г/сут & $207 \pm 77$ & $236 \pm 75$ & $239 \pm 68$ & $239 \pm 64$ & $<0,001$ & $<0,001$ & $<0,001$ \\
\hline Сахара, г/сут & $130 \pm 56$ & $133 \pm 49$ & $125 \pm 43$ & $113 \pm 37$ & 1,00 & 0,050 & $<0,001$ \\
\hline ПВ, г/сут & $26,3 \pm 12,4$ & $23,4 \pm 7,7$ & $20,9 \pm 6,0$ & $18,5 \pm 5,3$ & $<0,001$ & $<0,001$ & $<0,001$ \\
\hline Натрий, г/сут & $4,3 \pm 1,5$ & $4,5 \pm 1,4$ & $4,3 \pm 1,3$ & $4,1 \pm 1,2$ & 0,001 & 1,00 & 0,001 \\
\hline Белки, \% & $14,3 \pm 2,2$ & $13,7 \pm 1,8$ & $13,2 \pm 1,7$ & $12,7 \pm 1,7$ & $<0,001$ & $<0,001$ & $<0,001$ \\
\hline Жиры, \% & $46,5 \pm 6,7$ & $44,7 \pm 5,5$ & $43,1 \pm 5,6$ & $41,4 \pm 5,9$ & $<0,001$ & $<0,001$ & $<0,001$ \\
\hline Углеводы, \% & $37 \pm 6,8$ & $40 \pm 5,7$ & $42 \pm 6,0$ & $45 \pm 6,3$ & $<0,001$ & $<0,001$ & $<0,001$ \\
\hline Caxapa, \% & $22,8 \pm 5,6$ & $22,0 \pm 4,9$ & $21,6 \pm 5,0$ & $20,5 \pm 4,7$ & 0,003 & $<0,001$ & $<0,001$ \\
\hline
\end{tabular}

Таблица 3. Доля лиц с МС и отдельными компонентами МС в квартилях ГИ питания у женщин

\begin{tabular}{|c|c|c|c|c|c|}
\hline \multirow{2}{*}{ Компоненты МС } & \multicolumn{4}{|c|}{ Квартили ГИ } & \multirow{2}{*}{$\begin{array}{c}p^{*} \\
1-4\end{array}$} \\
\hline & 1 & 2 & 3 & 4 & \\
\hline MC (BHOK, 2009), \% & 62,0 & 61,7 & 63,0 & 61,5 & 0,792 \\
\hline MC (NCEP ATP III, 2001), \% & 30,0 & 31,5 & 30,8 & 31,0 & 0,643 \\
\hline MC (JIS, 2009), \% & 47,4 & 46,9 & 47,0 & 45,5 & 0,392 \\
\hline Ожирение (талия >80 см), \% & 79,2 & 77,6 & 77,7 & 75,2 & 0,025 \\
\hline Ожирение (талия >88 см), \% & 56,5 & 55,3 & 55,9 & 54,6 & 0,391 \\
\hline АГ (АД > 130/85 мм рт. ст.), \% & 70,7 & 70,5 & 73,6 & 73,2 & 0,200 \\
\hline Гипо-а-холестеринемия (<50 мг/дл), \% & 9,7 & 12,8 & 11,5 & 10,3 & 0,671 \\
\hline Повышенный уровень ХС ЛПНП (>115 мг/дл), \% & 87,1 & 85,6 & 87,0 & 85,8 & 0,574 \\
\hline Гипертриглицеридемия ( $\geq 150$ мг/дл), \% & 25,9 & 28,4 & 29,4 & 26,5 & 0,771 \\
\hline Гипергликемия (> 5,6 ммоль/л), \% & 21,1 & 18,2 & 16,5 & 19,4 & 0,339 \\
\hline
\end{tabular}

Примечание. * - значимость различий между 1 и 4 квартилями ГИ определяли методом $\mathrm{X}^{2}$

выше по сравнению с максимальным квартилем - 12,7\% и 41,4\% соответственно. И наоборот, доля углеводов была значимо ниже в 1 квартиле по сравнению с 4 квартилем, $37,2 \%$ и 45,0\% соответственно. Величина пищевых волокон (ПВ) в минимальном квартиле ГИ была значимо выше по сравнению с 4 квартилем, 26,3 г/сут и 18,5 г/сут соответственно. Потребление натрия в 1 квартиле ГИ было выше по сравнению с 4 квартилем, 4,3 г/сут и 4,1 г/сут соответственно (табл. 2).

Представляет интерес оценка возможной связи ГИ питания с риском развития МС. Доли лиц в 1 и 4 квартилях ГИ с наличием МС по критериям ВНOK (2009) значимо не различались. Также отсутствовало различие между крайними квартилями ГИ лиц с МС по критериям NCEP ATP III, 2001 и JID, 2009 (табл. 3). Отношение шансов (OR) MC по всем 3 использованным в оценке критериям MC в 4 квартиле ГИ относительно 1 квартиля ГИ питания у женщин 45-69 лет также не было значимым (табл. 4).

Важным диагностическим критерием МС является абдоминальное ожирение. Согласно критериям ВНОК (2009), абдоминальное ожирение диагностируется при величине окружности талии $>80$ см. В нашем обследовании доля лиц с абдоминальным ожирением в макси- мальном квартиле ГИ (75,2\%) была значимо ниже в сравнении с минимальным квартилем ГИ (79,2\%). При оценке величины абдоминального ожирения по критериям МС (NCEP ATP, III, 2001) (талия >88 cм) доли лиц с ожирением между крайними квартилями значимо не различались. Также отсутствовало значимое различие между крайними квартилями ГИ процента лиц по таким критериям МС, как АГ, гипертриглицеридемия, гипо-а-холестеринемия, повышенный уровень ХС ЛПНП и гипергликемия (см. табл. 3).

При оценке ГИ питания в зависимости от степени ожирения у женщин в квартилях ИМТ величина ГИ в группе с нормальной массой тела $\left(\right.$ ИМТ $\left.=23,3 \mathrm{kг} / \mathrm{M}^{2}\right)$ составила 53,6 единиц, в группе с максимальным уровнем ИМТ (37,3 кг/м²) - 52,7 единиц, т.е. имелось небольшое, но значимое снижение величины ГИ $(p<0,001)$. Различие в ЭЦ питания между крайними квартилями ИМТ не было значимым, но ЭЦ в расчете на 1 кг массы тела у женщин с ожирением (4 квартиль) была в 1,6 раза ниже по сравнению с нормой (1 квартиль). В максимальном квартиле доля (\%) белков и жиров в рационе была значимо выше, а углеводов - ниже по сравнению с минимальным квартилем, но эта разница была невелика. 
Таблица 4. Отношение шансов наличия МС в квартилях ГИ питания у женщин

\begin{tabular}{lcccc}
\hline & \multicolumn{3}{c}{ Квартили Ги } \\
\cline { 2 - 5 } & $\mathbf{1}$ & $\mathbf{2}$ & $\mathbf{3}$ & $\mathbf{4}$ \\
\hline OR MC (BHOK, 2009) & 1,00 & 0,988 & 1,043 & 0,977 \\
ДИ (95\%) & & 0,$832 ; 1,174$ & 0,$877 ; 1,239$ & 0,$823 ; 1,161$ \\
OR MC (NCEP ATP, 2001) & 1,00 & 0,$894 ; 1,285$ & 0,$866 ; 1,245$ & 1,048 \\
ДИ (95\%) & & 0,978 & 0,$874 ; 1,257$ \\
OR MC (JIS, 2009) & 1,00 & 0,$827 ; 1,157$ & 0,$832 ; 1,163$ & 0,928 \\
ДИ (95\%) & & & 0,$784 ; 1,097$ \\
\hline
\end{tabular}

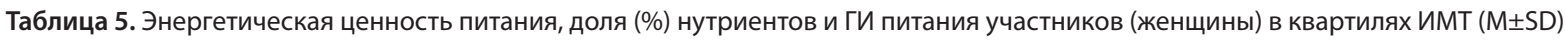

\begin{tabular}{|c|c|c|c|c|c|c|c|}
\hline \multirow{3}{*}{ Показатели } & \multicolumn{4}{|c|}{ Квартили ГИ } & \multicolumn{3}{|c|}{$\mathbf{P}$} \\
\hline & 1 & 2 & 3 & 4 & $1-2$ & $1-3$ & $1-4$ \\
\hline & $n=1099$ & $n=1099$ & $n=1100$ & $n=1099$ & & & \\
\hline 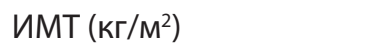 & $23,3 \pm 2,0$ & $27,6 \pm 0,9$ & $31,0 \pm 1,1$ & $37,3 \pm 4,1$ & $<0,001$ & $<0,001$ & $<0,001$ \\
\hline $\min -\max$ ИМТ & $16,02-25,97$ & $26,00-29,24$ & $29,25-33,09$ & $33,09-55,19$ & & & \\
\hline гИ & $53,6 \pm 3,7$ & $52,8 \pm 4,0$ & $53,3 \pm 3,8$ & $52,7 \pm 4,0$ & $<0,001$ & 0,586 & $<0,001$ \\
\hline Возраст, лет & $56,2 \pm 7,2$ & $58,0 \pm 6,9$ & $58,6 \pm 7,2$ & $58,2 \pm 7,0$ & $<0,001$ & $<0,001$ & $<0,001$ \\
\hline Масса тела, кг & $59,3 \pm 6,6$ & $69,4 \pm 5,7$ & $77,7 \pm 6,4$ & $92,5 \pm 11,8$ & $<0,001$ & $<0,001$ & $<0,001$ \\
\hline ЭЦ, ккал/день & $2366 \pm 693$ & $2339 \pm 662$ & $2311 \pm 669$ & $2312 \pm 680$ & 1,00 & 0,362 & 0,379 \\
\hline ЭЦ/кг массы тела, & $40,3 \pm 12$ & $33,9 \pm 9,9$ & $29,8 \pm 8,6$ & $25,4 \pm 8,0$ & $<0,001$ & $<0,001$ & $<0,001$ \\
\hline \% белков & $13,3 \pm 1,9$ & $13,5 \pm 2,0$ & $13,5 \pm 1,9$ & $13,8 \pm 2,1$ & 0,079 & 0,039 & $<0,001$ \\
\hline \% жиров & $43,2 \pm 6,2$ & $43,7 \pm 6,1$ & $44,0 \pm 6,2$ & $44,6 \pm 6,2$ & 0,261 & 0,008 & $<0,001$ \\
\hline \% углеводов & $42,2 \pm 6,9$ & $41,5 \pm 6,8$ & $41,1 \pm 6,8$ & $40,2 \pm 6,8$ & 0,073 & 0,001 & $<0,001$ \\
\hline
\end{tabular}

Важно отметить, что соотношение жиров и углеводов у женщин с ожирением в максимальном квартиле ИМТ не соответствовало рекомендациям по питанию, т.е. было несбалансированным. Несбалансированный тип питания также был и в группе с нормальной величиной ИМТ (табл. 5).

В исследуемой выборке женщин (45-69 лет) не было найдено связи ГИ фактического питания с наличием метаболических нарушений.

Эти данные отличаются от результатов оценки связи ГИ питания и МС у мужчин, жителей Новосибирска, возраст 45-69 лет, у которых было показано наличие значимой связи МС с ГИ. При этом доля мужчин с МС и абдоминальным ожирением (ВНОК, 2009) была существенно ниже, в среднем $36 \%$ и 44\% соответственно [20] по сравнению с женщинами в представленном исследовании.

\section{выводы}

1. ГИ питания у женщин в среднем составил 53 единицы и был близок к ГИ питания женщин в западных странах.

2. Фактическое питание, при различном значении ГИ у женщин (45-69 лет) г. Новосибирска, является несбалансированным - высокая доля жиров и низкая доля углеводов и не соответствует рекомендациям, в том числе в группе женщин с высоким показателем ИМТ.
3. Показано отсутствие значимой связи МС с ГИ питания, что, возможно, связано с высокой долей лиц в исследуемой выборке с МС (BНОК, 2009) и с абдоминальным ожирением, в среднем $62 \%$ и $77 \%$ соответственно.

4. В квартилях ИМТ у женщин величина ГИ была значимо ниже (на 0,9 единицы) в группе с ожирением по сравнению с группой с нормальным ИМТ. Важным изменением в питании у женщин, которое может оказывать положительное влияние на избыточную массу тела, было снижение в 1,6 раза величины калорийности питания в расчете на 1 кг массы тела в группе с ожирением по сравнению с контрольной группой. При этом общая калорийность питания во всех квартилях ИМТ значимо не различалась.

Информация о финансировании и конфликте интересов. Авторы заявляют об отсутствии явных и потенциальных конфликтов интересов, связанных с публикацией настоящей статьи.

Информация о финансировании. Проект HAPIEE поддержан грантами Wellcome Trust, UK 064947/Z/01/Z;081081/Z/06/Z; National Institute of Aging, USA (1R01 AG23522).

Выражаем благодарность д.М.Н., проф. Малютиной С.К., руководителю проекта НAPIEE.

Участие авторов. Кунцевич А.К. - сбор и обработка материала, анализ данных, написание текста; Мустафина С.В. - написание текста; Веревкин Е.Г. - формирование базы данных, анализ; Щербакова Л.В. формирование базы данных, анализ; Рымар О.Д. - концепция и дизайн исследования. 


\section{СПИСОК ЛИТЕРАТУРЫ | REFERENCES}

1. Jenkins D.J., Wolever T.M., Taylor R.H. et al. Glycemic index of foods: a physiological basis for carbohydrate exchange. Am. J. Clin. Nutr. 1981; 34(3): 362-366.

2. Atkinson FS, Foster-Powell K, Brand-Miller JC. International Tables of Glycemic Index and Glycemic Load Values: 2008. Diabetes Care. 2008:31(12):2281-2283. doi: 10.2337/dc08-1239.

3. Разина А.О., Ачкасов Е.Е., Руненко С.Д. Ожирение: современный взгляд на проблему // Ожирение и метаболизм. - 2016. - Т. 13. - №1. - C. 3-8. [Razina AO, Achkasov EE, Runenko SD. Obesity the modern approach to the problem. Obesity and Metabolism. 2016;13(1): 3-8. (in Russ)] doi: 10.14341/omet201613-8

4. Лобыкина Е.Н., Колтун В.С̧., Хвостова О.И. Гликемический индекс продуктов и использование его в диетотерапии ожирения // Bonp питания. - 2007. - T. 76. - №1. - C. 14-21. [Lobykina YeN., Koltun VS, Hvostova Ol. Food glycemic index and its application in the dietetics of obesity. Problem of Nutrition. 2007;76(1):14-21 (in Russ)]

5. Мустафина С.В., Малютина С.К., Рымар О.Д., Щербакова Л.В., M. Bobak, Воевода М.И. Эпидемиология ожирения и развитие нарушений углеводного обмена по данным проспективного исследования в Сибири. Ожирение и метаболизм. - 2015. -Т. 12 - № 4. C. 24-28. [Mustafina S.V., Malyutina S.K., Rymar O.D., Shcherbakova L.V., Bobak M., Voevoda M.I. The epidemiology of obesity and the development of disorders of glucose metabolism according to a prospective study in Siberia. Obesity and Metabolism. 2015; 12(4): 24-28 (in Russ)] doi: 10.14341/OMET2015424-28

6. Кунцевич А.К. Риск метаболического синдрома и питание населения. Ожирение и метаболизм. - 2015. - Т. 12 -№ 1. С. 3-10 [Kuntsevich AK. The risk of metabolic syndrome and nutrition. Obesity and Metabolism. 2015; 12(1): 3-10 (in Russ)] doi: 10.14341/ omet201513-10

7. Blaak EE, Antoine JM, Benton D, et al. Impact of postprandial glycaemia on health and prevention of disease. Obes. Rev. 2012;13(10):923984. doi: 10.1111/j.1467-789X.2012.01011.x.

8. Mathews MJ, Liebenberg L, Mathews EH. How do high glycemic load diets influence coronary heart disease? Nutr. Metab. 2015;12(1):6. doi: 10.1186/s12986-015-0001-x.

9. Barclay AW, Petocz P, McMillian-Price J, et al. Glycemic index, glycemic load, and chronic disease risk - a meta-analysis of observational studies. Am. J. Clin. Nutr. 2008; 87(3): 627-637.

10. McKeown NM, Meigs JB, Liu S, et al. Carbohydrate Nutrition, Insulin Resistance, and the Prevalence of the Metabolic Syndrome in the Framingham Offspring Cohort. Diabetes Care. 2004;27(2):538-546. doi: 10.2337/diacare.27.2.538.
11. Pi-Sunyer FX. Glycemic index and disease. Am. J. Clin. Nutr. 2002; 76 (1): 290S-298S.

12. Рекомендации экспертов Всероссийского научного общества кардиологов по диагностике и лечению метаболического синдрома (второй пересмотр). М.: BHOK; 209. [Recommendations of experts of Russian Society of Cardiology on diagnosis and treatment of metabolic syndrome (second revision). Moscow: VNOK; 2009. (in Russ)]

13. Executive Summary of the Third Report of the National Cholesterol Education Program (NCEP) Expert Panel on Detection, Evaluation and Treatment of High Blood Cholesterol in Adults (Adult Treatment Panel III). JAMA. 2001; 285 (19): 2486-2497.

14. Alberti KGMM, Eckel RH, Grundy SM, et al. Harmonizing the Metabolic Syndrome: A Joint Interim Statement of the International Diabetes Federation Task Force on Epidemiology and Prevention; National Heart, Lung, and Blood Institute; American Heart Association; World Heart Federation; International Atherosclerosis Society; and International Association for the Study of Obesity. Circulation. 2009:120(16):1640-1645. doi: 10.1161/circulationaha.109.

15. Mendez MA, Covas MI, Marrugat J, et al. Glycemic load, glycemic index, and body mass index in Spanish adults. The American Journal of Clinical Nutrition. 2009;89(1):316-322. doi: 10.3945/ajcn.2008.26444.

16. Rossi M, Bosetti C, Talamini R, et al. Glycemic index and glycemic load in relation to body mass index and waist to hip ratio. Eur. J. Nutr. 2010;49(8):459-464. doi: 10.1007/s00394-010-0104-0.

17. Flood A., Peters U., Jenkins D.J. et al. Carbohydrate, glycemic index, and glycemic load and colorectal adenomas in the Prostate, Lung, Colorectal, and Ovarian Screening Study // Am. J. Clin. Nutr. 2006; 84 (5): 1184-1192.

18. Wen W, Shu XO, Li H, et al. Dietary carbohydrates, fiber, and breast cancer risk in Chinese women. The American Journal of Clinical Nutrition. 2009;89(1):283-289. doi: 10.3945/ajen.2008.26356.

19. Nakashima M, Sakurai M, Nakamura K, et al. Dietaey glycemic index glycemic load and blood lipid levels in middle-aged Japanese men and women. J. Atheroscler. Thromb. 2010; 17(10): 1082-95.

20. Кунцевич А.К., Симонова Г.И., Мустафина С.В., и др. Гликемический индекс рационов фактического питания и риск развития метаболического синдрома у мужского городского населения Новосибирска (популяционное исследование) // Бюллетень сибирской медицины. - 2016. - Т.15. - №4. - C. 67-76. [Kuntsevich AK, Simonova GI, Mustafina SV, et al. The glycemic index diets and the risk of metabolic syndrome in male urban population of Novosibirsk (population studies). Bulletin of Siberian Medicine. 2016; 15(4): 67-76 (in Russ)] doi: 10.20538/1682-0363-2016-4-67-76

\section{ИНФОРМАЦИЯ ОБ АВТОРАХ [AUTHORS INFO]}

*Кунцевич Александр Константинович, к.б.н. [Alexander K. Kuntsevich, PhD]; адрес: Россия, 630089, Новосибирск, ул. Бориса Богаткова, д. 175/1 [address: 175/1, B. Bogatkova street, Novosibirsk, 630089, Russia]; ORCID: http://orcid.org/0000-0002-2191-1706; eLibrary SPIN: 1688-6325; e-mail: akkun2006@rambler.ru

Мустафина Светлана Владимировна, д.м.н. [Svetlana V. Mustafina, ScD]; eLibrary SPIN: 8395-1395; e-mail: svetamustafina@rambler.ru.

Веревкин Евгений Георгиевич, к.б.н. [Evgeniy G. Verevkin, PhD]; eLibrary SPIN: 8914-8917; e-mail: ewer@ngs.ru. Щербакова Лилия Валерьевна, С.H.C. [Liliya V. Shcherbakova]; ORCID: http://orcid.org/0000-0001-9270-9188; eLibrary SPIN: 5849-7040; e-mail: 9584792@mail.ru.

Рымар Оксана Дмитриевна, д.М.н., професcop [Oksana D. Rymar, ScD, professor]; eLibrary SPIN: 8345-9365; e-mail: orymar23@gmail.com

\section{ЦИТИРОВАТЬ:}

Кунцевич А.К., Мустафина С.В., Веревкин Е.Г., Щербакова Л.В., Рымар О.Д. Анализ фактического питания женского населения Новосибирска в зависимости от величины гликемического индекса их рациона // Ожирение и метаболизм. - 2018. — Т.15. — № 2 - C. 23-28. doi: 10.14341/OMET9293

\section{TO CITE THIS ARTICLE:}

Kuntsevich AK, Mustafina SV, Verevkin EG, Shcherbakova LV, Rymar OD. Analysis of the actual nutrition of the female population of Novosibirsk, depending on the magnitude of the glycemic index of their diet. Obesity and metabolism. 2018;15(2):23-28. doi: 10.14341/OMET9293 